\title{
3D SURVEY OF PRE-HISPANIC WALL PAINTING WITH HIGH RESOLUTION PHOTOGRAMMETRY
}

\author{
G. Lucet \\ Instituto de Investigaciones Estéticas, Universidad Nacional Autónoma de México, Méx. D.F., 04510, Mexico - \\ lucet@gmail.com
}

KEY WORDS: High Resolution, Photogrammetry, Cultural Heritage, 3D Surveying, Painting, Las Higueras

\begin{abstract}
:
The survey and representation of pre-hispanic wall painting use to be done with traditional photography, we describe the difficulties and limitations found in this approach and we show another solution to improve the quality of this documentation. It relies on the use of photogrammetry and MicMac program. The calculated orthophotos have an accurate geometry, they are high resolution and the three-dimensional models present a high level of detail. We obtain a complete representation which satisfy the requirements of art historians and conservators to study the meanings of the paintings and their conservation state. Furthermore, as this improvement is achieved by following a particular strategy for the photo sessions and the mathematical processing on the images, it doesn't need the acquisition of additional equipment.

We explain how we applied the method in the registration of a structure covered with pictorial representations that was discovered in the archaeological site of Las Higueras, México.
\end{abstract}

\section{INTRODUCTION}

Pre-Hispanic mural painting is the memory of important cultures that disappeared with the conquest of America and from which we know very little, that's why it get a particular importance that explains the effort done for its registration. We have few documents about these ancient cultures, only 14 preHispanic codex have survived to the fire of the spanish priest, they correspond to a period close to the conquest, therefore they don't cover a wide range of time nor the different mesoamerican cultural groups, their concerns focus primarily on astronomical data collection and the offering that had to be given to the gods on specific days of the calendar. In contrast, from all the mesoamerican area and period of time, we have many buildings covered with pictorial representations which make an invaluable documentation that different specialists study to know this disappeared world. They have found that the paintings contain elements of writing, dates and representations that describe religious ceremonies and concepts, important historical events, legends, scenes of daily life, way of dressing and adorning, among others. Thus, rather than being an artistic expression, the paintings are a primary source of information for various research disciplines and a priceless historiographical document. Unfortunately, these documents are fragile and difficult to maintain. The buildings on which they were painted crumble over time, the stucco are detached from their support because of environmental conditions, the sun, the rain and drought cycles, they fall down, the paint layers and their representation disappear for ever. Because of the amount of existing painting and the complexity of the possible solutions for safeguarding, the rate of deterioration exceeds financial possibilities and technical knowledge, institutions for cultural heritage conservation fail to control the situation and until now, there are no solutions to safeguard this heritage (Figure 1).

Therefore, 20 years ago, it was decided at the University (UNAM) to begin the project "Pre-Hispanic Mural Painting en México" to record and study the paintings that still survive in situ, and the one preserved in museums and in the cellars of the National Institute of Anthropology and History of Mexico (Pintura Mural Prehispánica, 2013). To date, 50,000 analog photographs and 60,000 digital have been captured, and 10 books produced with this information.
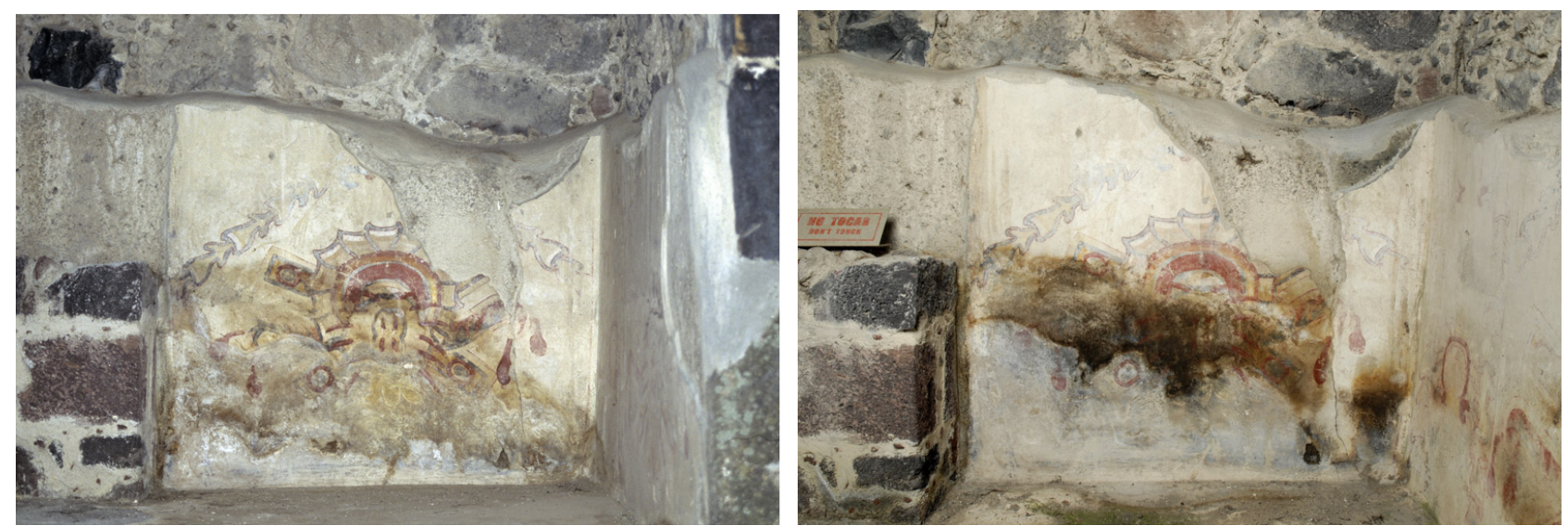

Figure 1: Teotihuacan mural, same painting taken with a gap of 4 years. (Photographic Archive of the "Pre-Hispanic Mural Painting in Mexico" project) 
The painting survey has important background, mainly as a need for restoration and conservation of easel paintings located in museums. Several initiatives and research have developed sophisticated methods to ensure high quality register of this information. The "Vasari" project developed by the National Gallery of London in 1989 (Saunders, 1993) for scanning of paintings and in 1999 the European project "CRISATEL: Conservation Restoration Innovation for image capture and digital Archiving to Enhance Training, Education and Long Life Learning" (Cotte, 2003; Ribes, 2003; Ribés, 2003) are good examples of this search, the accuracy obtained and technological advances that have occurred during the ten years that separate them. Solutions for museums can use robot systems to capture images perfectly parallel to the paint, they move the sensor accurately, without vibration, vertically and horizontally, in order to take the multiple photographs needed to obtain very high resolution images after the mosaicing of the photographs. The Vasari project uses grayscale images and a lighting system with 7 color filters to restore the color, in contrast the following projects rely on ultra-high definition multi-spectral scanner with an homogeneous lighting system. The current trend is to use the multispectral definition to go further than the color precision and to obtain material definition from this register (Remondino, 2011).

In the Vasari project, after the assembly of the images, the resolution was between 10 and 40 pixels per millimeter, and in the CRISATEL project, the digital image is limited by the size of the scanner sensor, which is 12,000 pixels by 30,000 lines per scan, which allows a resolution of $30 \mathrm{dots} / \mathrm{mm}$ in paintings of 1 $\mathrm{x} 1$ meter and up to $100 \mathrm{dots} / \mathrm{mm}$ for a small area paint.

Beyond the multispectral register of information, the need to get $3 \mathrm{D}$ representation was expressed because the deformation of support is essential in conservation studies and interventions (Guidi, 2002; Remondino, 2011), and for perception studies which include the understanding of behavior of light on the surface (Wei, 2013).

Because of the wide technical solutions and particular conditions of different kind of objects, specific equipment can be built to find an appropiate response to a situation (Taylor, 2001) and solutions may combine several digitization techniques such as high definition cameras, color laser and 3D optical scanner (Lahanier, 2008).

For the survey of pre-Hispanic wall painting, we are far from being able to work in fully controlled conditions. Besides economic conditions which always limit the work related to the world of culture, major constraints are imposed by the environment conditions and the location of paintings. Most of the time, we work outside or in tombs or tunnels, i.e. in environments characterized by dust, high temperature and humidity levels. Furthermore, archaeological sites may be located in isolated locations with difficult access. These conditions prevent the use of delicate or fragile equipment, with high energy requirements and we must find a technical solution with light weight equipment, easy to transport, resistant to movement, heat and moisture.

The "pre-Hispanic Mural Painting in Mexico" project records the painting, seeking the best quality that can be reach with photographic cameras, initially analog and digital since a few years. Sometimes, spectrophotometric data are taken to get additional information. A Macbeth ColorChecker card is used to register the color deformation and when the murals are large, multiple pictures are taken, close to the surface, to maintain a suitable resolution. Photographers try to take pictures parallel to the mural plane by tilting the camera, they do it in an empirical way, sometimes rails are used to control the movement of the equipment, but this solution is difficult to install in uneven floors. When assembling the mosaic of images in order to get orthophotos, the deformation becomes noticeable. That's why we are always searching for better ways of surveying the painting and architecture of this cultural heritage.

As we have seen previously, upgradable categories are: the spectrum (UV, EI, X-ray), color, levels of detail and geometric accuracy. Without new purchase, nor sacrificing the resolution we get today, the only part we can improve, is the geometric precision using digital photogrammetry. We can continue with the same procedure for color registration, trying to ensure an uniform illumination over the entire object and without changes throughout the acquisition phase of the images.

The level of detail is directly linked to the resolution of the representation and should be sub-millimetric. Given the difficult conditions of fieldwork and the magnitude of the murals, we found satisfactory a resolution of 3-4 pixels per millimeter of paint. Even if it is not a very high resolution, it provides a fairly complete information and it is a realistic solution.

The murals combine the characteristics of a painting with an architectural or natural support which is rarely flat, so in most cases, they present a complex morphology. The slopes and the vaults are recurring architectural elements in pre-Hispanic

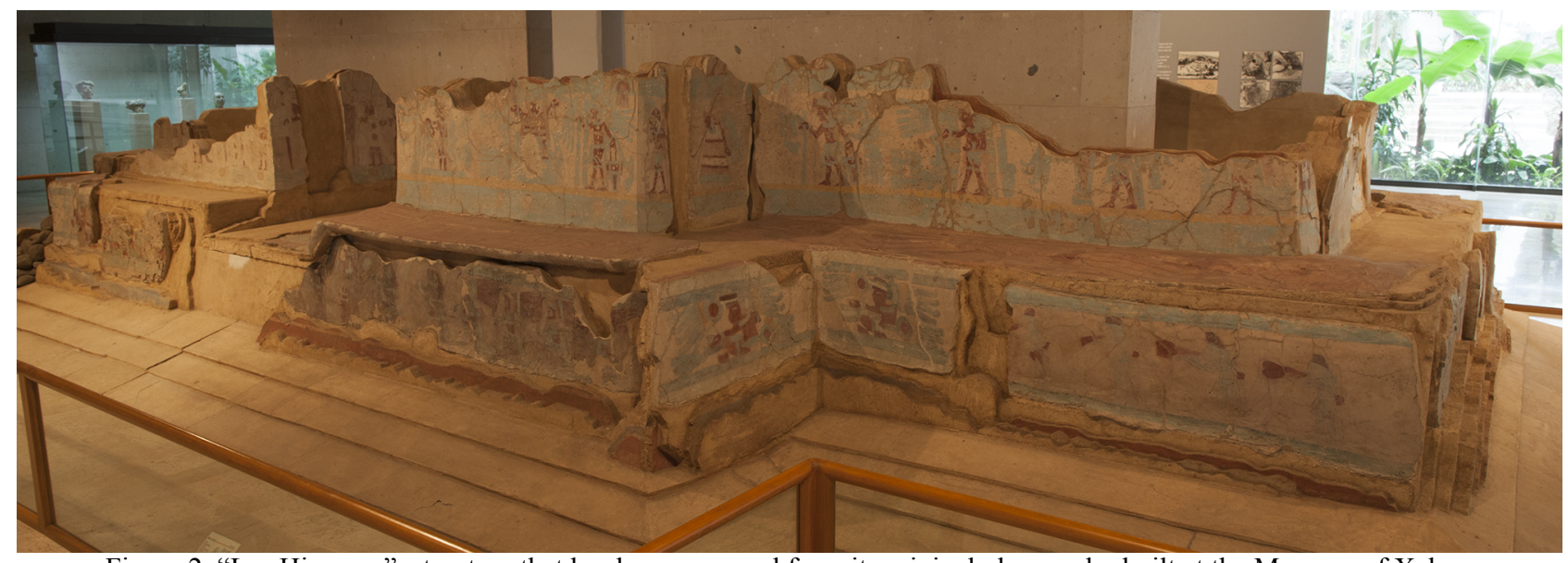

Figure 2. "Las Higueras", structure that has been removed from its original place and rebuilt at the Museum of Xalapa 
architecture and in the case of tombs, most of the time the paintings follow the natural shapes of stones. To these initial construction characteristics, we must add the aging process which has warped surfaces leaving very complex nonplanar surfaces. Sometimes, the surface has been covered by several layers of murals and because of partial detachment of some of the top layers, what is seen corresponds to different depths of paintings.

So, the projection of the representation to a plane is a complex problem and it may be a poor solution that dramatically reduces the original information when the surface is uneven. If we want to have a complete definition of the mural, retaining all the complexity of initial object in its current state of conservation, the only solution lies in the development of a three-dimensional definition and representation of the painting.

\section{DEVELOPMENT}

\subsection{Description of the case study}

To check if photogrammetry is a feasible solution for recording pre-Hispanic mural painting, we applied it to a building that was detached from its place of origin, the archaeological site of Las Higueras, state of Veracruz in Mexico, and was reconstructed in the Museum of Xalapa (Figure 2). It was a cross-shaped room located at the top of a pyramid. In pre-Hispanic times, the maintenance applied to the structure consisted in putting new layers of stucco covering the previous one, and we have counted the overlapping of at least thirty, some of which were completely painted, mainly on the upper temple. The inability to preserve the paintings in situ led archaeologists and restorers to decide to remove them, and to separate these different layers. This temple is now rebuilt at ground level for its exhibition at the museum, the most interesting pictorial phase was selected and in the destroyed or missing parts, the lower layers are visible. Peripheral walls of the construction have two sections, the inferior is slightly inclined and protruding with respect to the superior wall which is vertical. These two parts and the horizontal surface that lies between them were fully painted, as well as the door jambs. Overall, the murals measure approximately 60.4 linear meters and its area is around 36.25 square meters, for the orthophoto representation, we divided the set into 28 blocks to represent the walls and 6 for the floors.

The museum ensures stable environmental conditions, although the lighting is not entirely uniform and causes a significant change in color. We were not allowed to remove peripheral rails that prevent visitors to approach the paintings, this made the work more complicated and at the same time it is an additional argument that justifies the use of photogrammetry to obtain orthophotos, as it is not possible to take pictures in a position parallel to the surface.

\subsection{Images acquisition}

The photographic survey was made with a Canon EOS 5D Mark II camera of 21.1 Mpx. The first step consisted of an overall shooting all around the structure for a comprehensive definition of the building with a $24 \mathrm{~mm}$ lens. To generate the orthophotos, we divided the mural length by sections and in each position we got a set of 5 converging pictures with a $50 \mathrm{~mm}$ lens. In some cases, due to the spatial conditions, a $100 \mathrm{~mm}$ was used. We took 5 photographs to calculate the corresponding camera calibration of each optical configuration (lens type, object distance and focal length) and, in order to model color distortion, in each wall, we took a picture with the Macbeth
ColorChecker card. Altogether, we got 964 pictures, nevertheless, two thirds were used.

We also took references with a total station to scale the model, to relate it to a coordinate system and to check the occuracy of the result.

\subsection{Processing}

The image processing was done using MicMac (see reference), a free open-source software developed initially as an aerial photogrammetric tools and widely used for architectural survey (see reference TAPENADE).

The command sequence to generate orthophotos and point clouds begins with the referencing of the photographs in an absolute coordinate system with the following sequence:

- the search for pixels that connect the different pictures, "tiepoints" as they are called in MicMac documentation, with the command Tapioca.

- the mathematic modeling of each optical configuration, with Tapas.

- the calculation of the relative orientation of the 56 general photographs, with Tapas.

- the transformation of the model from the relative orientation to a global coordinate system, using the coordinates taken with the total station, with GCPBascule.

- the integration of the 510 photographs taken with the 50 and $100 \mathrm{~mm}$ lens and that so far are only linked by "tiepoints", to images that have been oriented in the absolute coordinate system, with Campari. The average residual was 0.67 pixels.

Finally, it's recommended to visualize the 3D representation of the pictures and tiepoints (with AperiCloud), that allows to check if no important mistake has been done up to this point (Figure 3).

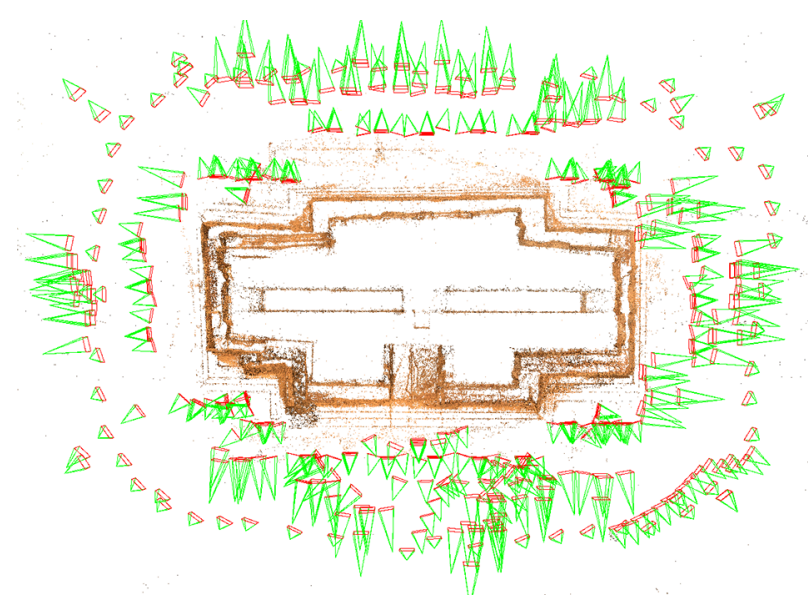

Figure 3. Displaying the position of the pictures

At this stage of the process, we have the spatial location of all the pictures and we can continue following two different strategies to generate the results, one for the orthophotos and the other to get the 3D model. 


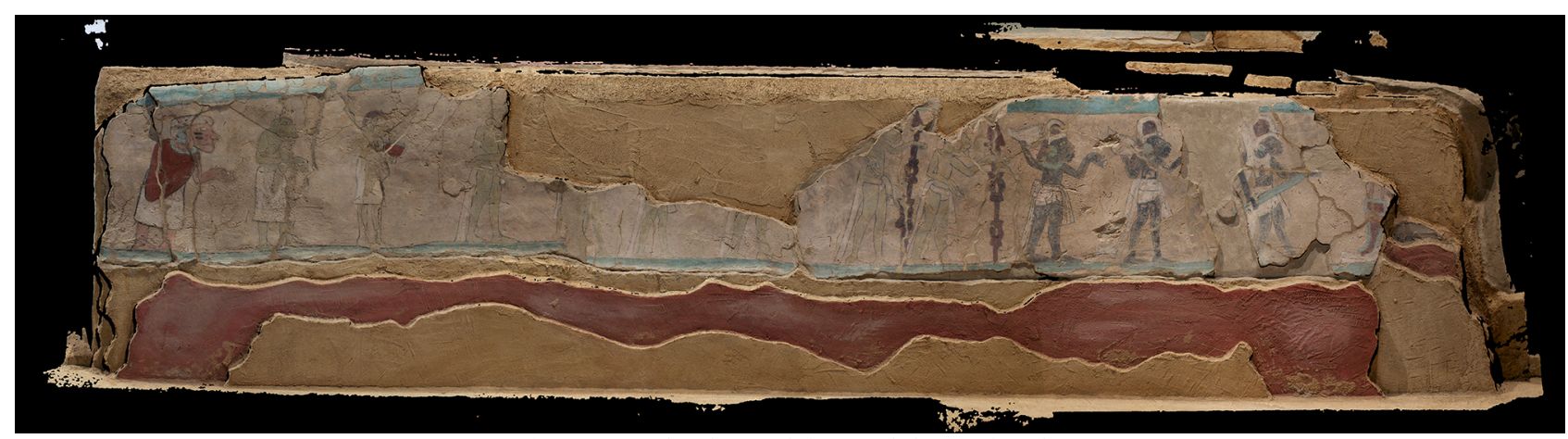

Figure 4. Orthophoto of the south lower façade.

In order to generate orthophotos:

- we calculated a local coordinate system which is perpendicular to the mural and we used an ortho-cylindrical reference system to avoid the problem of aligned cameras (with RepLocBascule). Masks were drawn on some pictures, they served as a reference to define the projection plane, calculating the average depth of points that correspond to the selected pixels.

- we created a mask using a rectified mosaic of the all surface with Tarama. That reduced the overall time of processing, limiting the calculation to the selected surface.

- at that point, it's possible to replaced the original CR2 files by tif files with color correction.

- the matching of the pixels of the different photographs was done with Malt Ortho and

- to obtain the orthophoto mosaicing with radiometric equalization, Tawny was used.

- to generate 3D point clouds orthophotos: Nuage 2Ply.

For the creation of the 3D point clouds of the whole structure: for each set of photographs, a master image was selected, it served as a reference for the pixel matching.

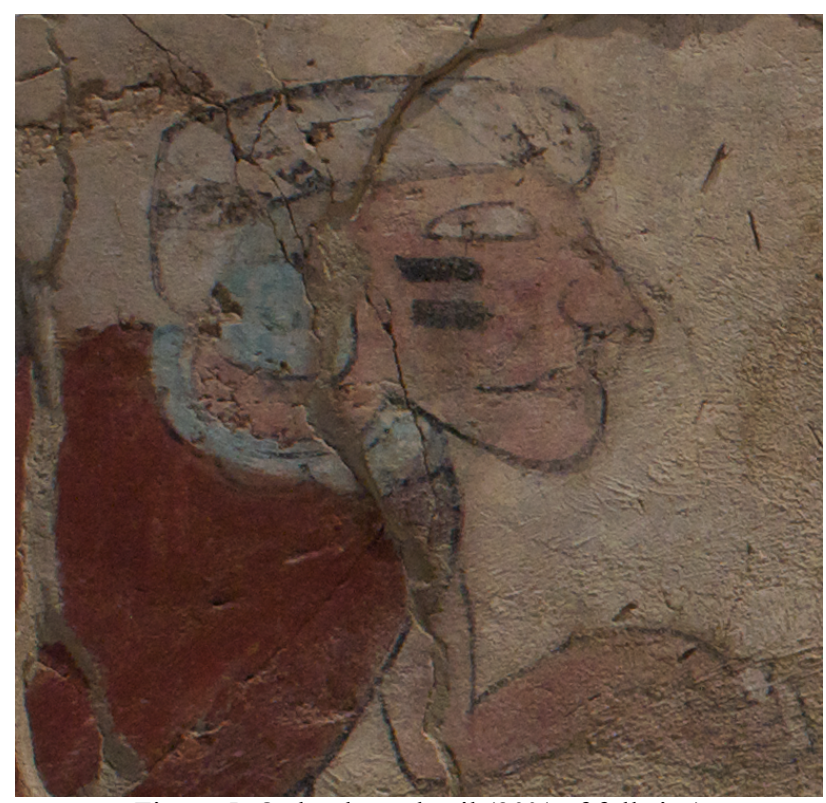

Figure 5. Orthophoto detail (30\% of full size)
- a mask was drawn in order to limit the calculation area.

- the image matching from color corrected images was done with the Malt GeomImage command

- point clouds were calculated with Nuage2Ply

\section{RESULTS AND DISCUSSION}

The obtained orthophotos are a projection onto a plane which corresponds to an average of the pixels defined by masks (Figure 4). So, they correspond to the best fitting plane of the

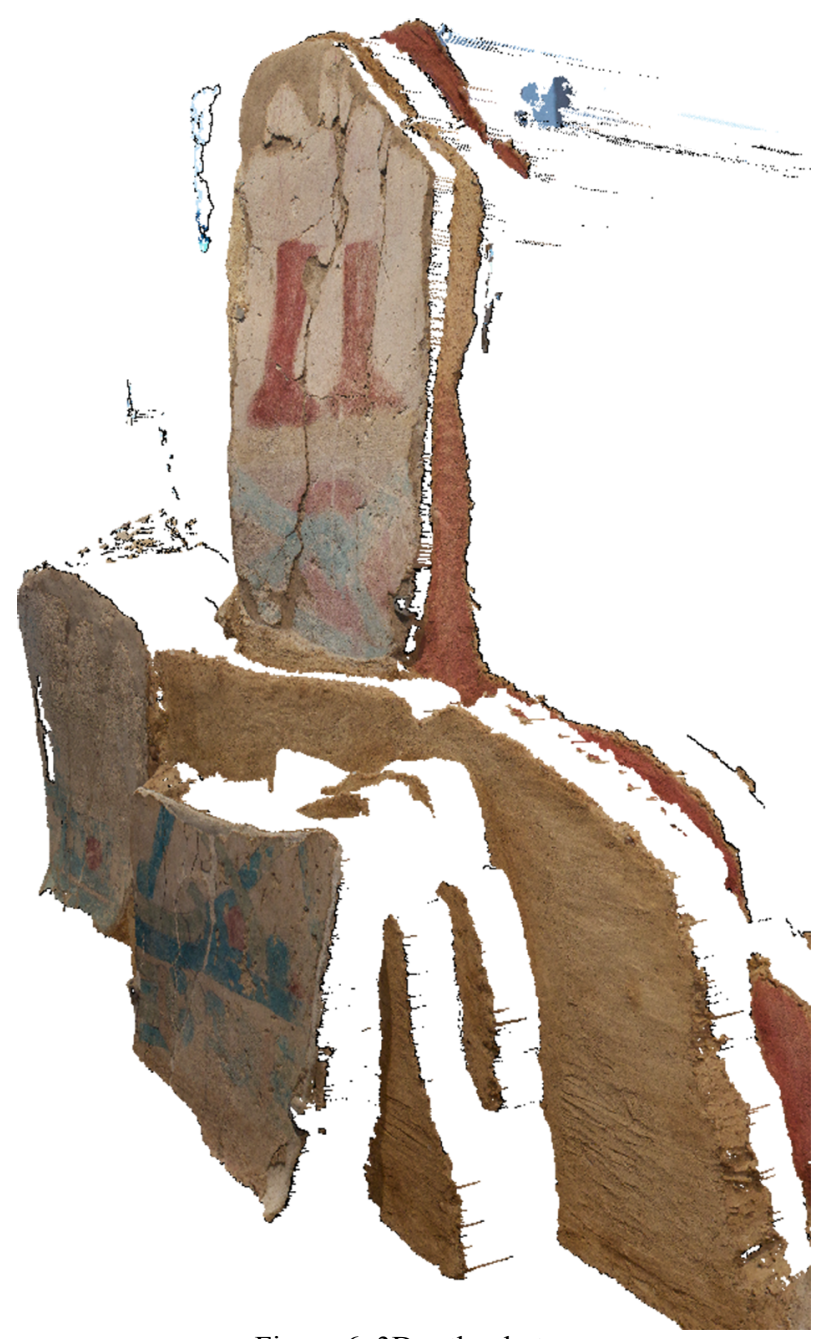

Figure 6. 3D orthophoto 


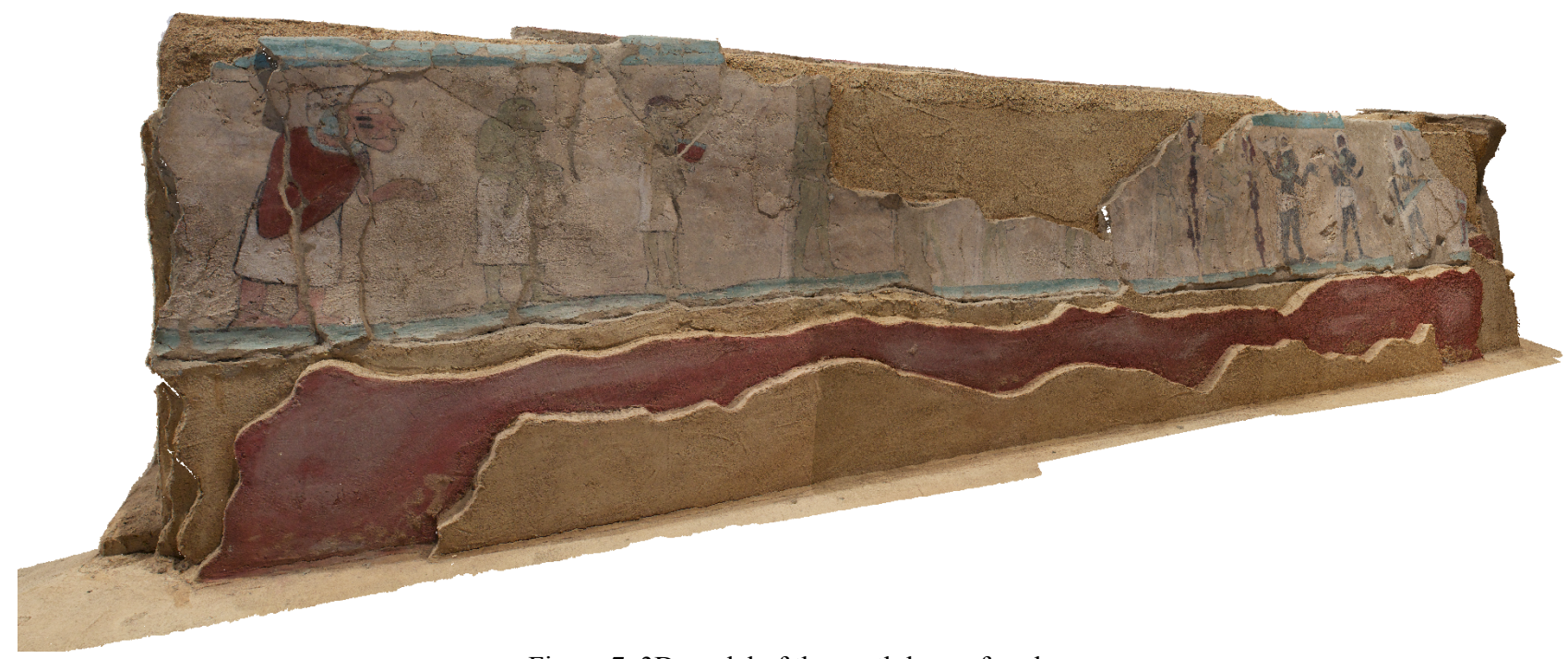

Figure 7. 3D model of the south lower façade

mural. To measure its accuracy, we compared the total station data with the corrected image. From a surface which measures $3.082 \mathrm{~m}$ long, on each end the difference between the measured and the orthophoto coordinates was 2 pixels or $0.48 \mathrm{~mm}$. The other six reference points showed the following differences: 2.7 , $0.87,2.2,0.68,1.3$, and $1.7 \mathrm{~mm}$. We would expect not to exceed a difference of $0.5 \mathrm{~mm}$, but at this time we can not say whether the problem comes from the measurement with the total station or from the photogrammetric process. According to Remondino (2011), for the correct survey of paintings and conservation treatment, accuracy between 0.1 and $1 \mathrm{~mm}$ should be expected, and for architecture, between 1 and $10 \mathrm{~mm}$. Overall, the result is very satisfactory and this difference is not significant to potentially generate misrepresentations in an historiographical analysis.

The color corresponds to what can be expected with an overall shot without lighting control and filters, when it is modeled only with a Macbeth ColorChecker card and registered in an RGB system. It's not bad for a general vision of the mural but it's not precise.

The average resolution of the orthophotos is around 4 pixels per millimeter $(\mathrm{GSD}=0.25 \mathrm{~mm})$ and it corresponds to the maximum that can be obtained from the original photographs. It's not as huge as what has been reported in museum projects for painting registration but it is close to the scanner resolution reported by Remondino (2011) and it is in the range of accepted resolution for researcher (Figure 5).

The 3D orthophotos present the benefit to be as precise as the orthophotos and to separate in space the different painting layers, it's an intermediate visualization between orthophoto and 3D model (Figure 6). It allows to appreciate the deformation of each layer of painting, as well as the correspondence between the different depths and the different figures.

Finally, the three-dimensional model calculated with photogrammetric method is the complete spatial definition of all the murals, with their gaps (Figure 7). Converted into a mesh, the visualization without color information clearly shows surface irregularities. We still get noise and we will work in its correction, moving parameters for the point cloud calculation or by further post-processing. Cracks and irregularities are accurately modeled. Our equipment didn't manage to load so large files and it was necessary to reduce the density of the meshes, sacrificing part of the 3D definition, this limitation will be overcome in the coming years by developments in hardware and software, and it doesn't mean a methodological error. Moreover, the reduction of the mesh does not imply a reduction of the information registered with color changes, since the texture applied to the geometry is extracted from the pictures.

\section{CONCLUSION}

With this proposal, we overcome many of the limitations we previously had in our way of recording the murals, we no longer need to carry special equipment to slide the camera along the mural plane, we only require to take many photographs following the rules imposed by the photogrammetric process. Even if we limit the evaluation of the result to the only generation of the orthophotos, we observe an improvement of the quality in terms of precision thanks to the precise modeling of the optical distortion, in addition we have to take in account that we got the flexibility to decide which is the projection plane that best fits the painting. Furthermore, the 3D orthophoto provides additional information about the different depths of the surface. Finally, we can create a full three-dimensional model of the construction, getting a complete $3 \mathrm{D}$ description of each mural with the irregularities, deformation, cracks, bulges and layers.

Importantly, these improvements in working methods and results can be done with the current equipment.

Regarding the application shown in this paper, we feel that the resolution could be improved, especially for orthophotos, this is easy and can be done with the same workflow we have followed, if the pictures were taken closer to the surface or with a higher resolution camera. 
The only method to fully and accurately record pre-Hispanic murals to get the information about the deformations of its support is by their three-dimensional modeling and digital photogrammetry manage to do it when it relocates each pixel in space and makes it a dot full of color. There are many advantages to this volumetric definition of the mural. For an historian interested in the study of the representation, in addition to the meaning and interpretation, this precise description is a tool to understand and explain some of the distortions of the figures, to better distinguish the original forms, he can also see if the artist took and used surface deformations to carry out his work. Ambiguities are removed and elements that could be misleading and be registered as graphics, can clearly appears as surface irregularities. The restorer gets the deformation of the support of the painting, and this is a substantial assistance to understand the process of deterioration that occurs, to monitor the evolution of the object over time and define conservation strategies.

We believe that digital photogrammetry is the ideal tool to get a true representation of the pre-Hispanic mural, because of the nature of the fieldwork, the accuracy of the result and the different possible outputs.

\section{REFERENCES}

\section{References from Journals:}

Guidi, G. et al., 2004. Painting Survey by 3D Optical Scanning: The Case of "Adoration of the Magi" by Leonardo da Vinci. Studies in Conservation, 49(1), pp. 1-12.

Remondino F. et al., 2011. Review of geometric and radiometric analyses of paintings. Photogrammetric Record, 26(136), pp. 439-461.

\section{References from Other Literature:}

Cotte, P. \& Duopuy, M., 2003. CRISATEL High Resolution Multispectral System. In PICS 2003, Image Quality, Image Capture Systemes Conference, pp.161-165.

Ribes, A. et al., 2003. Color and Multispectral Imaging with the CRISATEL. In Multispectral System. Is and ts Pics Conference, (Conf 56), pp. 215-219.

Ribés Cortés, A., 2003. Analyse multiespectrale et reconstruction de la réflectance spectrale de tableaux de maître. Docteur. France: Ecole Nationale Supérieure des Télécommunications.

Robson, S. et al., 2004. Periodic photogrammetric monitoring and surface reconstruction of a historical wood panel painting for restoration purposes. In International Archives of the Photogrammetry, Remote Sensing and Spatial Information Sciences, 35(B5), pp. 395-400.

Saunders, David \& Cupitt, John, 1993. Image Processing at the National Gallery: The VASARI Project. National Gallery technical bulletin. Volume 14, 1993. London: National Gallery Publications.

Taylor, J., Beraldin, J.-A. \& Godin, G., 2001. 3D Imaging Collaboration Between the National Research Council of Canada and European Museums and Cultural Organizations. In Electronic Imaging and the Visual Arts. Montréal, Québec, Canada, October 3-5.

\section{References from websites:}

Lahanier, C. et al., 2008. Two-dimensional multi-spectral digitization and three-dimensional modelling of easel paintings. http://nparc.cisti-icist.nrc-cnrc.gc.ca/npsi/ctrl?action=shwart\& index $=$ an\&req $=8914123 \&$ lang $=$ en $($ April 15, 2013).

MicMac, n.d., http://www.micmac.ign.fr (Sept 10, 2012).

Pintura Mural Prehispánica, Bienvenido a Pintura Mural Prehispanica | Pintura Mural Prehispanica http://www.pinturamural.esteticas.unam.mx/ (April 8, 2013).

TAPENADE, Tools and Acquisition Protocols for Enhancing Artifacts Documentation, http://www.tapenade.gamsau.archi.fr/TAPEnADe/Home.html (Sept 10, 2012)

Wei, W., de Tagle, A. \& Hummelen, I., 2005. Three dimensional documentation of two dimensional works of art [5857-08]. In The International Society for Optical Engineering. Optical Methods for Arts and Archaeology. Boston, USA, pp. 52-61.

\section{ACKNOWLEDGEMENTS}

I want to thank Irais Hernandez for her work correcting the colors of the photographs, preparing masks and editing the material.

This research was supported by the PAPIIT program of DGAPA-UNAM (IT400213) and by the project "La Pintura Mural Prehispánica en México".

Thanks to the IGN, Institut Géographique National, and in particular to Marc Pierrot Deseilligny for its great work developing the free opensource sofware MicMac. 